\title{
The Oriented and Flux-Weighted Current Density Stagnation Graph of LiH
}

\author{
R. J. F. Berger ${ }^{1, *}$ and M. Dimitrova ${ }^{2}$ \\ ${ }^{1}$ Fachbereich für Chemie und Physik der Materialien, Paris-Lodron \\ Universität Salzburg, Jakob-Harringerstr. 2a, A-5020 Salzburg, \\ Österreich. \\ ${ }^{2}$ Department of Chemistry, Faculty of Science, P.O. Box 55, A. I. \\ Virtasen aukio 1, FI-00014 University of Helsinki, Finland. \\ ${ }^{*}$ Corresponding author: R. J.F. Berger, raphael.berger@plus.ac.at
}

January 3, 2022

A scheme is introduced to quantitatively analyze the magnetically induced molecular current density vector field $\mathbf{J}$. After determining the set of zero points of $\mathbf{J}$, which is called its stagnation graph (SG), the line intergals $\Phi_{\ell_{i}}=$ $-\frac{1}{\mu_{0}} \int_{\ell_{i}} \mathbf{B}_{\text {ind }} \cdot \mathrm{d} \mathbf{l}$ along all edges $\ell_{i}$ of the connected subset of the SG are determined. The edges $\ell_{i}$ are oriented such that all $\Phi_{\ell_{i}}$ are non-negative and they are weighted with $\Phi_{\ell_{i}}$. An oriented flux-weigthed (current density) stagnation graph (OFW-SG) is obtained. Since $\mathbf{J}$ is in the exact theoretical limit divergence free and due to the topological characteristics of such vector fields the flux of all separate vortices and neighbouring vortex combinations can be determined by adding the weights of cyclic subsets of edges of the OFW-SG. The procedure is exemplified by the case of LiH for a perpendicular and weak homogeneous external magnetic field $\mathbf{B}$. 


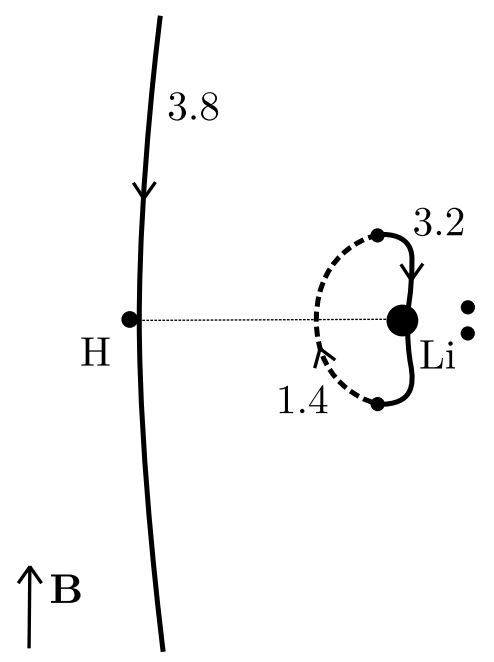

Oriented flux-weighted current density stagnation graph of $\mathrm{LiH}$, in the external magnetic field $\mathbf{B}$. Flux weights are given in $\mathrm{nA} / \mathrm{T}$.

\section{Introduction}

Any physical changes in molecules are ultimately triggered by the electromagnetic force. Molecules, hence, are restricted to interact with their environment via (not neccessarily small) electronic or magnetic perturbations. Therefore, the study of the molecuar magnetic response is of high relevance. In molecular magnetic response theory, the induced electonic current density $\mathbf{J}$ is a key quantity from which all other magnetic response properties can be calculated in a quasi-classical fashion by evaluating expectation value integral-like expressions. Hirschfelder has coined the term subobservable for such quantities.[1] A series of reviews on the current state of the research on this subject is available.[2-4] One branch of this research field is concerned with a topological and quantitative characterization of $\mathbf{J}$, an undertaking that can be seen in analogy to the topological characterization of the electron density. $[5,6]$

We have recently reported some progress on the quantitative characterization of $\mathbf{J}[7]$ which is, for example, often used in arguments about the possible aromatic or antiaromatic nature of a compound. For the quantification of the molecular current one is typically interested in the flux $\Phi$ through a particular surface $\mathcal{S}$ that is chosen by chemical intuition or according to other demands or model ideas, such as the "ring-current" model for (annelated) ring systems[2]. The idea underlying our recent work[7] was to use instead of the surface integral of $\mathbf{J}$ over $\mathcal{S}$ the line intergral of the corresponding induced magnetic field $\mathbf{B}_{\text {ind }}$ over the boundary line $\partial \mathcal{S}$ of the surface $\mathcal{S}$, that is, to apply the integral variant of the Ampère-Maxwell law,

$$
\Phi_{\partial \mathcal{S}}=\iint_{\mathcal{S}} \mathbf{J} \cdot \mathrm{d} \mathbf{s}=\frac{1}{\mu_{0}} \oint_{\partial \mathcal{S}} \mathbf{B}_{\text {ind }} \cdot \mathrm{d} \mathbf{l} .
$$

Below we employ the simple example of the lithium hydride $(\mathrm{LiH})$ molecule to show how 
this method can be naturally extended by applying it to the so called stagnation graph (i.e. the set of zero points) of $\mathbf{J}$, such that the quantification of separate current vortices becomes simple and automatizable.

\section{Discussion}

The topology (principle shape) of the magnetically induced current density $\mathbf{J}$ field of $\mathrm{LiH}$ has been discussed previously already in great detail by Stevens and Lipscomb (1964) [8], Keith and Bader (1993)[6] and later by Pelloni, Lazzeretti and Zanasi (2009)[9], so we will only give a summary here. ${ }^{1}$ Placing $\mathbf{B}$ parallel to the $z$ axis and perpendicular to the $\mathrm{Li}-\mathrm{H}$ bond (placed in $y$ direction) results in a $\mathbf{J}$ field that is composed of exactly two separate current vortices, each of them possessing a central stagnation line (see Fig. $1)$. Both vortices are separated from each other by a single closed surface $\mathcal{K}$ of the exact topology and the approximate geometry of a sphere. The spherical vortex domain (inner vortex) is completely enclosed in the other domain which extends over the remaining full molecular space (outer vortex). The outer vortex has an open stagnation line $\ell_{1}$ extending from $z=-\infty$ to $z=\infty$ and lying in the $y, z$ plane. $\ell_{1}$ is passing the $\mathrm{H}$ atom at a short distance of a fraction of an atomic unit and is bending towards it. Above and below the $x, y$ plane $\ell_{1}$ is bending slightly towards the center of the LiH molecule but straightens out at larger $\pm z$ heights. The current vortex around this stagnation line $\ell_{1}$ is diatropic, thus, according to the convention we use, clockwise oriented if the external field $\mathbf{B}$ is pointing upwards ( $=z$ direction). The approximately spherical inner vortex domain can be imagined as inserted in between the streamlines of the outer vortex which are bypassing this domain similarly to how a laminar-flowing fluid would pass near a ball submerged into it. This results in two isolated "toroidal" stagnation points $p_{+}, p_{-}$ on $\mathcal{K}$ where the outer flow diverges/converges in/from all directions on the sphere $\mathcal{K}$.

The inner domain is enclosing the $\mathrm{Li}$ atom but not the $\mathrm{H}$ atom. However the $\mathrm{Li}$ atom is geometrically not centered in $\mathcal{K}$ but shifted by a signficant distance towards the $\mathrm{H}$ atom. The topology of the inner current vortex is that of a toroidal flow, like the water flow of a waterspout fountain with an inner reflux tube. The inner reflux stream is closely passing the Li atom and roughly directed perpendicular to both $\mathbf{B}$ and the $\mathrm{Li}-\mathrm{H}$ bond, it is in the $x, y$ plane and double-s shaped and it contains a single separatrix line which connects the $(3,1)$ (i.e. a source critical point) point $p_{+}$with the $(3,-1)$ (i.e. a sink critical point) point $p_{-}$also inside $\mathcal{K}$.

The doughnut shaped dry region of the waterspout fountain corresponds to the central stagnation line $\ell_{2}$ of the inner vortex. This stagnation line is a topological circle in case of the $\mathbf{J}$ field and geometrically approximately D-shaped. Remarkably, the described toroidal current flow is aligned in the $y, z$ plane which means that the main direction of the inner flow (the reflux tube in the fountain metaphor) and the direction of the counter-directed outer flow (water pouring down) is $x$ (or $-x$ ) such that also $\ell_{2}$ lies in the $y, z$ plane. Consequently, $\ell_{2}$ is composed of two branches - one diatropic stagnation

${ }^{1}$ General proceedures to compute stagnation points and stagnation lines have been published as well and are freely available.[10, 11] 


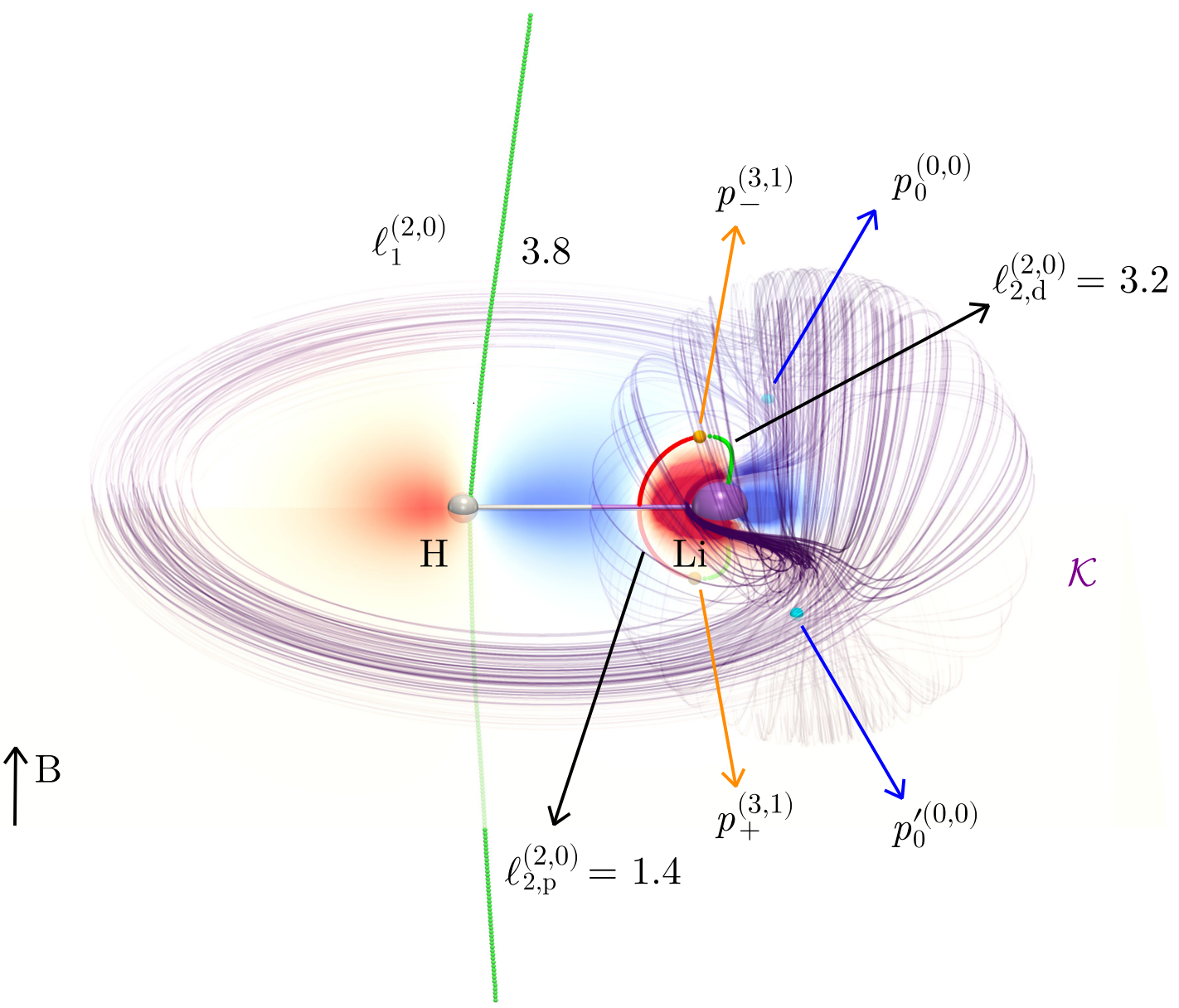

Figure 1: The magnetically induced current density field $\mathbf{J}$ of $\mathrm{LiH}$ in an external magnetic field $\mathbf{B}$ perpendicular to the bond, contains a set of stagnation points ( $=$ zero points): the vortex critical lines $\ell_{1}, \ell_{2, p}, \ell_{2, d}$ (in green or red) of (Jacobian) characteristics $(2,0)$, the branching points $p_{+}, p_{-}$of characteristics $(3,1)$ and $(3,-1)$ respectively (in orange), and the two isolated torus or source- and sink-critical points $p_{0}$ and $p_{0}^{\prime}$ (in cyan). Streamlines that are closely passing the torus critical points are embedded in a topological and geometrical approximate spherical surface $\mathcal{K}$, which separates the main molecular diatropic vortex around $\ell_{1}$ from the toroidal vortex around $\ell_{2}=\ell_{2, p} \cup \ell_{2, d} \cup\left\{p_{0}, p_{0}^{\prime}\right\}$. The flux $\Phi_{\ell_{1}}$ in the main vortex amounts to $3.8 \mathrm{nA} / \mathrm{T}$ while the toroidal vortex flux $\Phi_{\ell_{2}}=4.3(=3.2+1.4) \mathrm{nA} / \mathrm{T}$. The current density flux flowing away the viewer is illustrated in red, while the opposite flow is shown in blue.

line $\ell_{2, d}$ and one paratropic stagnation line $\ell_{2, p}$. They branch and recombine in so-called $(0,0)$ critical points $p_{0}$ and $p_{0}^{\prime}$ with all three eigenvalues of $\nabla \mathbf{J}$ equal to 0 . 
Since no currents can pass from one vortex (domain) into or out of another one, a total current flux can be assigned to each separate vortex. As we have shown previously[7] and since $\mathbf{B}_{\text {ind }}$ is vanishing at infinity in $z$ and $y$ direction, the total current flux of the outer vortex can be obtained from the line integral

$$
\Phi_{\ell_{1}}=-\frac{1}{\mu_{0}} \int_{\ell_{1}} \mathbf{B}_{\text {ind }} \cdot \mathrm{d} \mathbf{l}
$$

Here we choose the direction of the line $\ell_{1}$ such that $I_{\ell_{1}}$ becomes positive. In this way, every current-density stagnation graph can be uniquely oriented to yield a directed graph. Furthermore, to each "egde" (line segment) of a stagnation graph, the flux integral can be assigned such that an oriented edge-weighted graph with strictly positive weights is obtained.

To obtain the current flux of the toroidal current inside $\mathcal{K}$, one can make use of the notion that the full flux is passing through the D-shaped closed stagnation line $\ell_{2}$ (composed of $\ell_{2, d}$ and $\ell_{2, p}$ ), thus:

$$
\begin{aligned}
\Phi_{\ell_{2}} & =-\frac{1}{\mu_{0}} \oint_{\ell_{2}} \mathbf{B}_{\text {ind }} \cdot \mathrm{d} \mathbf{l} \\
& =-\frac{1}{\mu_{0}}\left(\int_{\ell_{2, d}} \mathbf{B}_{\text {ind }} \cdot \mathrm{d} \mathbf{l}+\int_{\ell_{2, p}} \mathbf{B}_{\text {ind }} \cdot \mathrm{d} \mathbf{l}\right) \\
& =\Phi_{\ell_{2, d}}+\Phi_{\ell_{2, p}}
\end{aligned}
$$

where again for each integral an orientation for each of $\ell_{2, d}$ and $\ell_{2, p}$ is obtained. A simple numerical intergration scheme has been applied (see ?? for details), and we have thereby obtained $\Phi_{\ell_{1}}=3.8, \Phi_{\ell_{2, d}}=3.2$ and $\Phi_{\ell_{2, d}}=1.4 \mathrm{nA} / \mathrm{T}$. The corresponding surface integrals of $\mathbf{J}$ over the $y, z$ plane in the region left of $\ell_{1}$ and over the surface enclosed by $\ell_{2}$ yielded 4.0 , and $4.6 \mathrm{nA} / \mathrm{T}$, respectively, both in very good agreement with the corresponding values of the line intergals $(3.8 \mathrm{nA} / \mathrm{T}$ and $3.2+1.4=4.6 \mathrm{nA} / \mathrm{T})$. The details of these computations ate given in section ??.

We conjecture that any field $\mathbf{J}$ partitioned ${ }^{2}$ into vortices separated by surfaces of separatrices has a connected or disconnected set of stagnation graphs (together with a set of isolated stagation points). Then also for every such vortex, a cyclic subgraph defining the vortex, like the pair of stagnation lines in the example of the toroidal vortex in $\mathrm{LiH}$, exists. Setting this cyclic subgraph to $\partial \mathcal{S}$ in the line integral in equation (1) then gives the current flux in this vortex.

\footnotetext{
${ }^{2} i$. e. decomposable into pairwise disjunct sets and where the union is the complete set
} 


\section{Conclusion and Outlook}

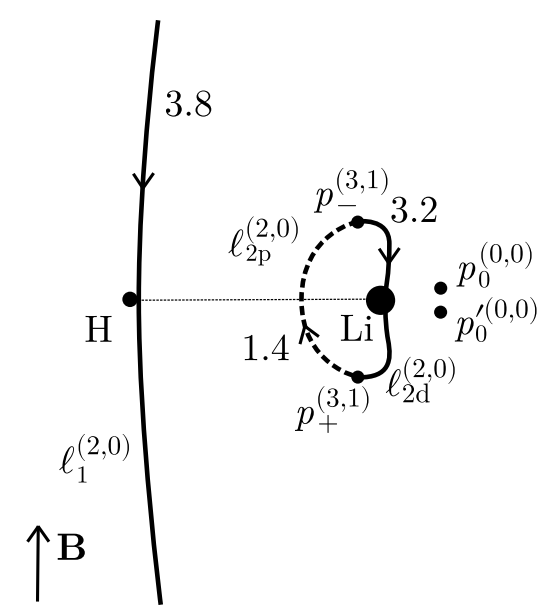

Figure 2: An oriented flux-weighted current density stagnation graph (OFW-SG) of LiH, with two connected subsets $\left(\ell_{1}\right.$ and $\left.\ell_{2 d} \cup \ell_{2 p} \cup\left\{p_{0}, p_{0}^{\prime}\right\}\right)$ and two isolated stagnation points $\left(p_{+}, p_{-}\right)$. Vortex current density weights obtained by integration of the Ampère law expression are given in units of $\mathrm{nA} / \mathrm{T}$ ).

The presented approach effectively gives a complete magnetically induced current flux analysis of a given molecule. It can be achieved by evaluating the line integral from Eq. (1) for each "edge" of the stagnation graph (and thereby, orienting all edges). The results of a OFW-SG can be condensed into a schematic digramm like it is shown in Fig. 2 and the strategy is completely generalisable to non-planar and non-cyclic molecules, and can be carried out for any molecule with a non-trivial connected stagnation graph. It has been described in the literature that large molecules without symmetry elements may not posses a non-trival stagnation graph, meaning that $\mathbf{J}$ cannot be partitioned into smaller non-trivial simple vortices, or at least only barely so. This is usually the case if there are significant current contributions parallel to the external field $\mathbf{B}$ which cause the vortices to show a helical component, neccessarily leading to non-zero current transfer in between otherwise separate vortices. For such cases, instead of $\mathbf{J}$, a pseudo-J field can be investigated where the parallel component is projected out. One then obtains a pseudo stagnation graph $[12,13]$ for which the same procedure as above can be applied. We are currently investigating this possibility.

\section{Acknowledgements}

MD thanks the Finnish Cultural Foundation for the research grant. Computational resources were provided by the Finnish Grid and Cloud Infrastructure (persistent identifier urn:nbn:fi:research-infras-2016072533) and the CSC - IT Center for Science, Finland. 


\section{References}

[1] J. O. Hirschfelder, The Journal of Chemical Physics 1978, 68, 5151-5162.

[2] P. Lazzeretti, Progress in Nuclear Magnetic Resonance Spectroscopy 2000, 36, 1-88.

[3] D. Sundholm, H. Fliegl, R. J. Berger, WIREs Computational Molecular Science 2016, 6, 639-678.

[4] D. Sundholm, M. Dimitrova, R. J. F. Berger, Chemical Communications 2021, 57, $12362-12378$.

[5] J. A. N. F. Gomes, Physical Review A 1983, 28, 559-566.

[6] T. A. Keith, R. F. W. Bader, The Journal of Chemical Physics 1993, 99, 3669-3682.

[7] R. J. F. Berger, M. Dimitrova, R. T. Nasibullin, R. R. Valiev, D. Sundholm, Physical Chemistry Chemical Physics 2022.

[8] R. M. Stevens, W. N. Lipscomb, The Journal of Chemical Physics 1964, 40, 2238 2247.

[9] S. Pelloni, P. Lazzeretti, R. Zanasi, Theoretical Chemistry Accounts 2009, 123, 353-364.

[10] G. Monaco, F. F. Summa, R. Zanasi, Journal of Chemical Information and Modeling 2020, 61, 270-283.

[11] T. J. P. Irons, A. Garner, A. M. Teale, Chemistry 2021, 3, 916-934.

[12] R. Carion, B. Champagne, G. Monaco, R. Zanasi, S. Pelloni, P. Lazzeretti, Journal of Chemical Theory and Computation 2010, 6, 2002-2018.

[13] G. Monaco, P. D. Porta, M. Jabłoński, R. Zanasi, Physical Chemistry Chemical Physics 2015, 17, 5966-5972. 